\title{
A High Quantum Efficiency High Readout Speed 1024 Pixel Ultraviolet-Visible-Near Infrared Waveband Photodiode Array
}

\author{
Rihito Kuroda ${ }^{\dagger}$ (member), Takahiro Akutsu ${ }^{\dagger}$, Yasumasa Koda ${ }^{\dagger}$, \\ Kenji Takubo ${ }^{\dagger \dagger}$, Hideki Tominaga ${ }^{\dagger \dagger}$, Ryuta Hirose ${ }^{\dagger \dagger}$, \\ Tomohiro Karasawa ${ }^{\dagger \dagger}$ and Shigetoshi Sugawa ${ }^{\dagger}$ (member)
}

\begin{abstract}
A high QE and high readout speed ultraviolet-visible-near infrared (UV-VIS-NIR) light waveband linear photodiode array (PDA) is demonstrated in this paper. For the developed 1024 pixel PDA with the pixel size of $25 \mu \mathrm{m}^{\mathrm{H}} \times 2500 \mu \mathrm{m}^{\mathrm{V}}$, seven types of high transmittance optical layers were introduced for multiply divided pixel groups to achieve high QE for receiving light waveband and multiple transfer gates were placed along the long side of PD to improve readout speed. The fabricated PDA exhibited an average QE of $70 \%$ for 200-800 nm and $80 \%$ for $200-320 \mathrm{~nm}$ wavebands, the full well capacity (FWC) of over $70 \mathrm{pC}$ and the line scan period of 0.33 msec simultaneously. The condition of top surface dopant concentration of the surface $\mathrm{p}^{+}$layer of photodiode (PD) to prevent sensitivity degradation due to deuterium lamp irradiation was also clarified.
\end{abstract}

Keywords: linear photodiode array, optical layer, UV-VIS-NIR waveband, deuterium lamp.

\section{Introduction}

Spectrometers are widely used in the various fields of scientific analyses, life science, environmental assessment, food inspection and so on ${ }^{1) ~ 3)}$. High sensitivity for UV-VIS-NIR light waveband is strongly required for PDA used in spectrometers to analyze various measurement samples accurately. In addition, a high readout speed performance is beneficial to improve the measurement throughput. Furthermore, high stabilities of light sensitivity and dark current of PDA toward UV-light irradiation are required for a long time use of the spectrometers without complicated maintenance. The quantum efficiency (QE) of a light detector is determined by the product of light transmittance to the photoelectric conversion part of PD and the efficiency to collect photo-generated carriers. In recent years, a Si photodiode junction technology to achieve high incident light sensitivity and high stability toward UV-light using a surface high concentration layer with steep dopant concentration profile formed on

Received August 31, 2015; Revised December 14, 2015; Accepted February 3,2016

$†$ Graduate School of Engineering, Tohoku University (Sendai, Japan)

† Shimadzu Corporation

(Kyoto, Japan)

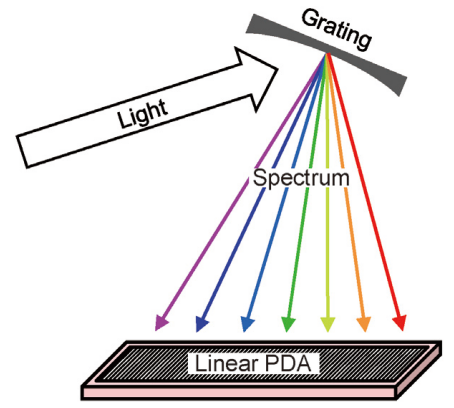

Fig.1 Schematic image of the typical detector of a spectrometer using PDA.

flattened Si surface has been developed, and single PD, PDA and area CMOS image sensors using the developed technology have been reported to exhibit superior characteristics ${ }^{4)}$ 9). Fig.1 shows the schematic image of a spectrometry system with a diffraction grating and a PDA as spectral light detector. Spectral light is irradiated to the pre-designated positions of the PDA. For general PDAs, the dielectric film above the PD is uniformly formed across the whole light receiving surface, thus, it is difficult to optimize the transmittance for all of the receiving light waveband.

Meanwhile, image sensors with Fabry-Perot spectral filters directly formed on the top surface of monochrome CMOS image sensors have been reported to be useful to form hyperspectral line scan imagers ${ }^{10)}$. The reported 
sensor contains different spectral filters arranged as a staircase-like structure covering rows of pixels. It has been proposed to be applied for line scanner with scanning function. As PDAs for spectrometers, a high sensitivity is to be achieved for a wide light waveband if each PD region contains a high transmittance optical layer corresponding to the pre-designated receiving light waveband. This concept has been proposed recently ${ }^{11) ~ 12)}$.

The shape of photodiode pixel of PDA is large rectangle in order to receive spectrum light from various optical configuration of spectrometers. However, the charge readout path is generally placed on the short side of the PD. This limits the signal readout speed. In order to improve the signal readout speed of the PDA with large rectangle PDs, PDAs with multiple transfer gates along the long side of PD have been developed ${ }^{6}$.

In this work, we demonstrate a 1024 pixel PDA for UV-VIS-NIR spectroscopy with high transmittance optical layers for multiply divided pixel groups for a high QE for UV-VIS-NIR waveband and multiple transfer gates along the long side of PD for a high readout speed. In addition, the high concentration surface $\mathrm{p}^{+}$layer with steep dopant concentration profile was introduced to improve Si incident light sensitivity and stability to UVlight irradiation. The condition of dopant concentration of the surface $\mathrm{p}^{+}$layer to prevent sensitivity degradation due to deuterium lamp irradiation is also experimentally clarified in this paper.

\section{PDA Design and Fabrication Technology}

Fig.2 shows the conceptual diagram of the proposed PDA structure to achieve high QE for wide waveband. Here, pixel region is divided into several groups each contains multiple number of PDs. For each group, high transmittance optical layer is formed above PDs which is optimized toward the waveband to be received. Fig.3 shows the cross sectional viewgraph of a $\mathrm{PD}$ with a high transmittance optical layer. For the developed PDA, pixel region was divided into seven groups having a band pass filter type optical layer each with high transmittance toward the corresponding receiving light waveband.

Fig.4 shows the circuit block diagram of the developed PDA. The pixel size is $25 \mu \mathrm{m}^{\mathrm{H}} \times 2500 \mu \mathrm{m}^{\mathrm{V}}$. This large $\mathrm{PD}$ height is beneficial to account for various types of spectrometers' optical configurations as well as to increase the FWC. Here a high FWC is advantageous for improving photon shot noise limited signal-to-noise ratio

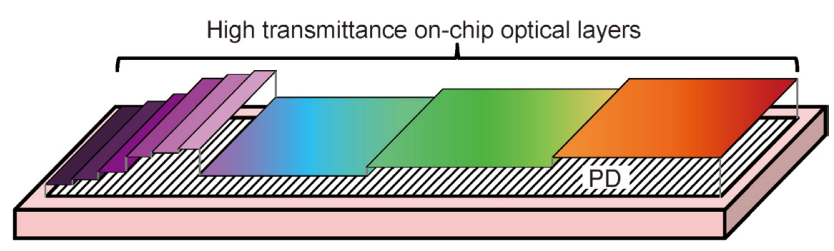

Fig.2 Conceptual diagram of the proposed PDA structure with optimum optical layers for multiply divided pixel groups.

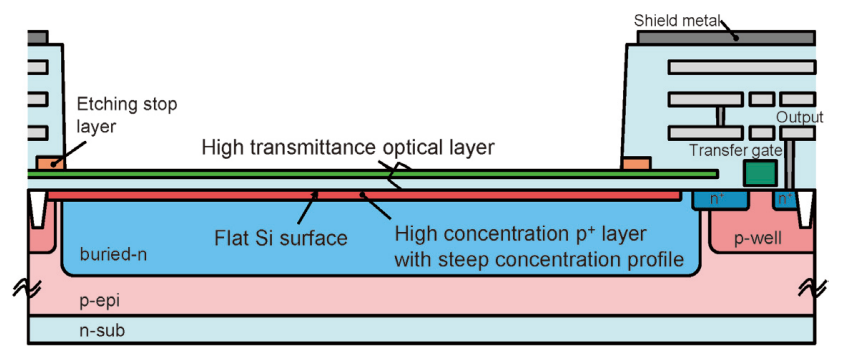

Fig.3 Cross sectional viewgraph of a PD with high transmittance optical layer. The surface pn junction technology enabling high sensitivity and high stability to UV-light was also introduced.

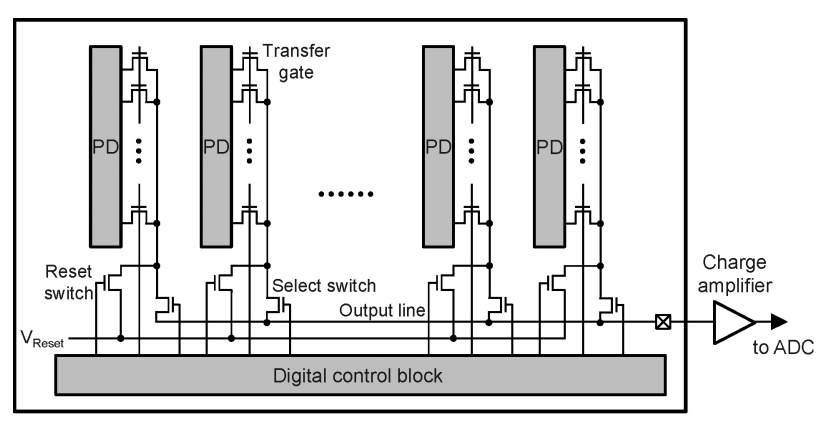

Fig.4 Circuit block diagram of the developed PDA. The pixel size is $25 \mu \mathrm{m}^{\mathrm{H}} \times 2500 \mu \mathrm{m}^{\mathrm{V}}$. Multiple numbers of transfer gates are placed along the long side of $\mathrm{PD}$.

(SNR) for absorption spectrometers that detect small changes of incident light levels due to absorption of target samples. For the PD, partially depleted buried pn junction was employed and the photo-generated charge is readout by charge amplifier placed outside the PDA chip. This PD structure is also beneficial to achieve a high FWC since the junction capacitance per unit area is larger in comparison to the fully depleted PD structure. For the PDA with partially depleted PD structure, the signal readout speed tends to be limited by the RC delay of PD. Thus, placing multiple numbers of transfer gates along the long side of each $\mathrm{PD}$ is effective to improve the signal readout speed by decreasing the RC delay ${ }^{6)}$. In this work, 18 transfer gates were placed along one of the long sides of each PD.

Fig.5 shows the chip fabrication process flow and Table 1 shows the detailed structure of the seven types of optical layers. For the formation of on-chip high transmittance multiple optical layers, several design 


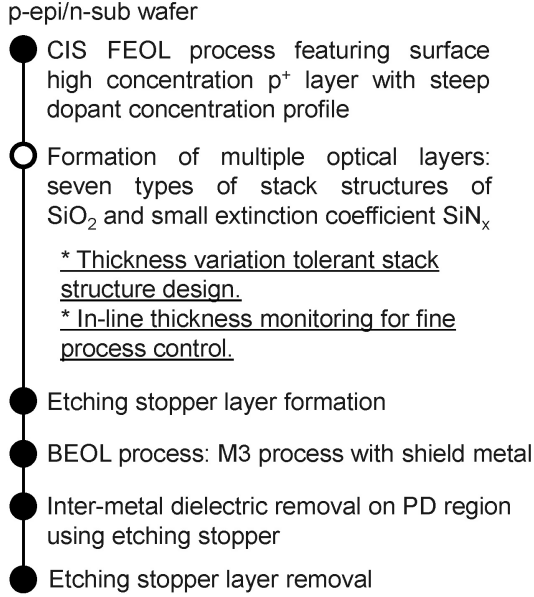

Fig.5 Chip fabrication process flow.

Table 1 Detail of the seven types of high transmittance optical layers formed on pixel region of the developed PDA.

\begin{tabular}{|c|c|c|c|c|c|c|c|c|c|c|c|}
\hline \multirow{2}{*}{\multicolumn{3}{|c|}{ Optical layer type }} & 1 & 2 & 3 & 4 & 5 & 6 & 7 & 1 & 3 \\
\hline & & & \multicolumn{3}{|c|}{ Two layers } & \multicolumn{3}{|c|}{ Four layers } & \multicolumn{3}{|c|}{ Two layers } \\
\hline \multirow{4}{*}{$\begin{array}{c}\text { Layer and } \\
\text { thickness } \\
\text { [nm] } \\
\text { (0th-layer: } \\
\text { Si) }\end{array}$} & 4 th & $\operatorname{SiN}_{\mathrm{x}}$ & - & - & - & 20 & 20 & 20 & - & - & - \\
\hline & 3 rd & $\mathrm{SiO}_{2}$ & - & $\cdot$ & $\cdot$ & 68 & 80 & 95 & - & $\cdot$ & - \\
\hline & $2 n d$ & $\operatorname{SiN}_{\mathrm{x}}$ & 20 & 20 & 20 & 10 & 10 & 10 & 20 & 20 & 20 \\
\hline & 1 st & $\mathrm{SiO}_{2}$ & 56 & 66 & 76 & 7 & 7 & 7 & 20 & 56 & 76 \\
\hline \multicolumn{3}{|c|}{$\begin{array}{l}\text { Receiving light } \\
\text { waveband [nm] }\end{array}$} & $\begin{array}{l}200- \\
209\end{array}$ & $\begin{array}{l}210^{-} \\
223\end{array}$ & $\begin{array}{l}224- \\
244\end{array}$ & $\begin{array}{l}245- \\
267\end{array}$ & $\begin{array}{l}268- \\
286\end{array}$ & $\begin{array}{l}287^{-} \\
316\end{array}$ & $\begin{array}{l}317 . \\
443\end{array}$ & $\begin{array}{l}444- \\
628\end{array}$ & $\begin{array}{l}629^{-} \\
1100\end{array}$ \\
\hline \multicolumn{3}{|c|}{ Pixel number } & $\begin{array}{c}1023^{-} \\
977\end{array}$ & $\begin{array}{l}976^{-} \\
954\end{array}$ & $\begin{array}{l}953^{-} \\
919\end{array}$ & $\begin{array}{l}918^{-} \\
881\end{array}$ & $\begin{array}{l}880^{-} \\
850\end{array}$ & $\begin{array}{l}849^{-} \\
801\end{array}$ & $\begin{array}{l}800- \\
595\end{array}$ & $\begin{array}{l}594- \\
298\end{array}$ & $\begin{array}{c}297^{-} \\
0\end{array}$ \\
\hline
\end{tabular}

and fabrication strategies were employed and they are summarized as below.

I. For materials of the optical layers above PDs, high integrity $\mathrm{SiO}_{2}$ and small extinction coefficient $\mathrm{SiN}_{\mathrm{x}}$ were employed. These materials have long been utilized for image sensors and LSI, thus beneficial to maintain the process simplicity and long term reliability of the optical layers. The small extinction coefficient $\mathrm{SiN}_{\mathrm{X}}$ film which is transparent to UV-light waveband down to at least $200 \mathrm{~nm}$ was formed by plasma CVD process with optimized mixed gas flow rate and deposition pressure ${ }^{13-14)}$.

II. $\mathrm{SiO}_{2} / \mathrm{Si}$ interface formed by direct oxidation of $\mathrm{Si}$ was applied for all the optical layer types to maintain the high quality interface ${ }^{5)}$. This is beneficial to reduce an increment of fixed charges due to UV-light exposure stress, resulting in an improvement of performance stability for a long time use of PDAs.
III. The thickness of the top surface layers of all the optical layers as well as the first and the second layers of optical layer types 4 6 were designed to be the same. This enables to apply the same films for different groups resulting in a reduction of process-induced thickness variation, leading to a reduction of transmittance variation.

IV. The designed optical layers are basically band pass filters. The transmittance at the boundary pixels of different pixel groups tend to be low. In addition, the process induced thickness variation may cause degradation of the transmittance especially at the boundary pixels. Thus, the boundaries of the different pixel groups must be carefully designed to maintain the overall high $\mathrm{QE}$ performance. In this work, the designed thickness differences of the optical layers; the first layers of the optical layer types 1 3 and 7 and third layers of the types $4 \sim 6$, were formed by the etching process of the initially formed same films. With the in-line film thickness monitoring explained next, this design is advantageous to achieve a high tolerance toward thickness variation of the optical layers due to the film deposition process. In addition, it is expected to be effective to form multiple optical layers within the same PD around the boundaries of the pixel groups.

V. For the fabrication process, the optical layer thickness was tuned carefully using the in-line layer thickness monitoring results obtained during a sequence of the film etching process.

About the Si wafer substrate and process flow to fabricate the PDA chip, Si wafer with low concentration $20 \mu$ m-thick p-type epitaxial layer on n-type substrate was employed to improve NIR light sensitivity. The surface high concentration $\mathrm{p}^{+}$layer with steep dopant concentration profile was formed on flattened Si surface to improve sensitivity and stability toward UV-light. The aforementioned high transmittance optical layers were formed before the metallization process. After the shield metal formation process, inter-metal dielectric film was removed on the $\mathrm{PD}$ regions by an etching process. In order to stop this dielectric film etching above the optical layers, an etching stopper layer was formed prior to the metallization process. Finally, the etching stopper layer was removed. The employed chip fabrication technology was based on a $0.18 \mu \mathrm{m}$ 1P3M CMOS process with buried partially depleted PD. 


\section{Fabricated PDA Chip Performance}

\subsection{Light Sensitivity and Readout Speed}

Fig.6 shows the micrograph of the fabricated 1024 pixel PDA chip ${ }^{12)}$. The die size is $28.6 \mathrm{~mm}^{\mathrm{H}} \times 3.3 \mathrm{~mm}^{\mathrm{V}}$. The multiple pixel groups with different optical layers are easily recognized by its outlook. For the comparison of QE performance, a PDA with $3.8 \mu \mathrm{m}$-thick uniform $\mathrm{SiO}_{2}$ film above all of the pixel region was also fabricated. In addition, for the comparison of signal readout speed, a PDA with single transfer gate on a short side of each PD was also fabricated with the same pixel size and process conditions.

Fig.7 shows the photoelectric conversion characteristic of the fabricated PDA. A good linearity and a high FWC of $73 \mathrm{pC}$ sufficient for absorption spectrometers were successfully obtained due to the large PD size with the buried partially PD structure. Fig.8 (a-b) shows the measured $\mathrm{QE}$ as a function of wavelength of the developed PDA and the reference sample with the $3.8 \mu \mathrm{m}$-thick $\mathrm{SiO}_{2}$ film above PD, i.e., the inter-metal dielectric of the three metal layers. For the developed PDA, the high QE performance was successfully obtained, i.e., the average and the minimum $\mathrm{QEs}\left(\mathrm{QE}_{\text {average }}\right.$ and $\mathrm{QE}_{\text {minimum }}$ ) were $70 \%$ and $50 \%$ for 200-800 nm waveband and $80 \%$ and $70 \%$ for $200-320$ $\mathrm{nm}$ waveband, respectively. In addition, the beat of $\mathrm{QE}$ curve due to the optical interference appeared for the reference sample was significantly reduced in the developed PDA. It is because the optical path lengths of the optical layers formed on the developed PDA are relatively short to the wavelength of the light receiving waveband. The obtained result is to be beneficial to improve spectral sensitivity variation due to the fluctuation of optical system of spectrometers. Fig.9 (a) shows the pixel driving pulse and (b-c) show the PD photo charge readout efficiency as a function of transfer gate on time. For this measurement, the light illumination level corresponding to the $90 \%$ of FWC was employed. In comparison to the conventional structure with single transfer gate on the short side of PD, over 100 times faster readout speed was achieved for the developed PDA with 18 transfer gates on one of the long sides of PD. The obtained improvement of signal readout speed was well matched with the estimation using the RC delay calculation. The high FWC and high signal readout speed performances were successfully achieved by the fabricated PDA.

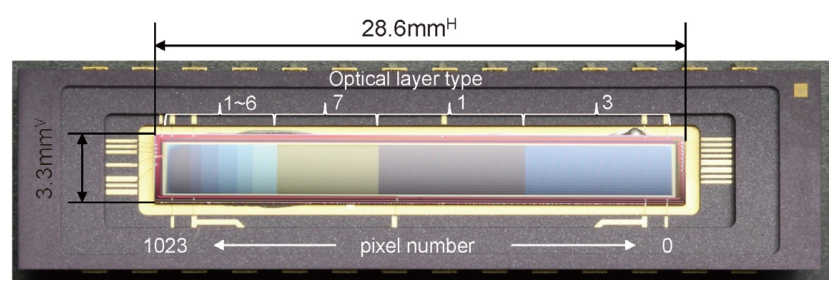

Fig.6 Micrograph of the fabricated PDA chip with high transmittance optical layers for multiply divided pixel groups.

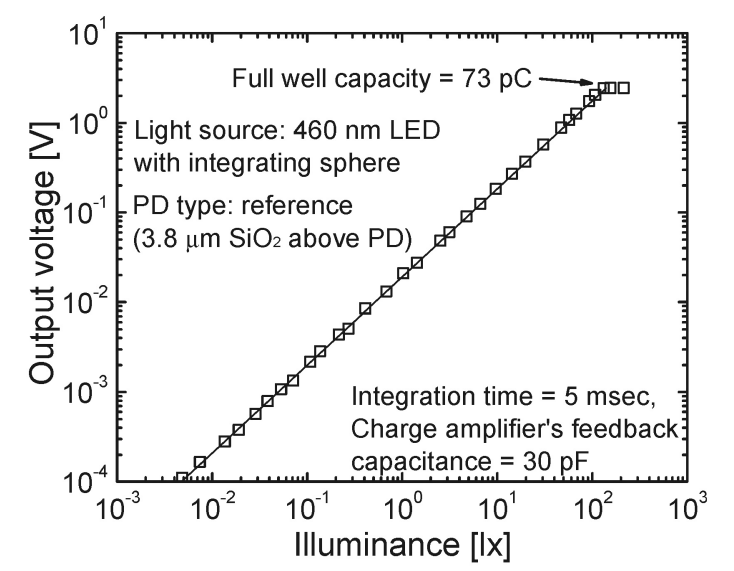

Fig.7 Photoelectric conversion characteristic of the fabricated PDA.

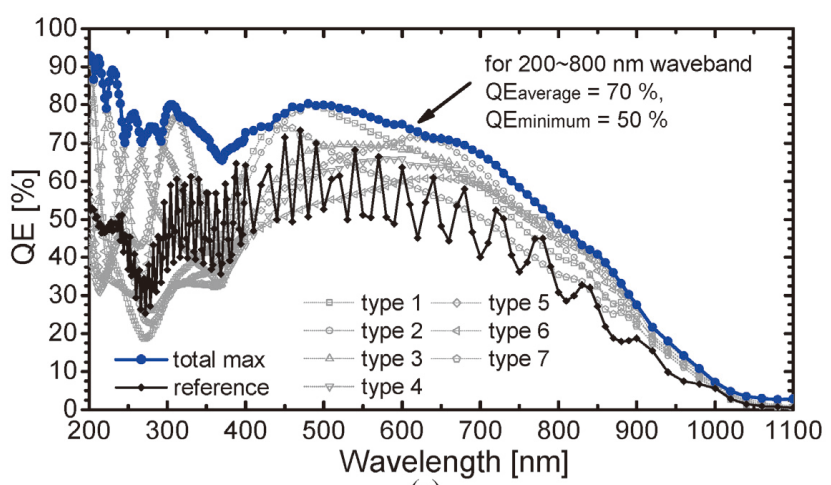

(a)

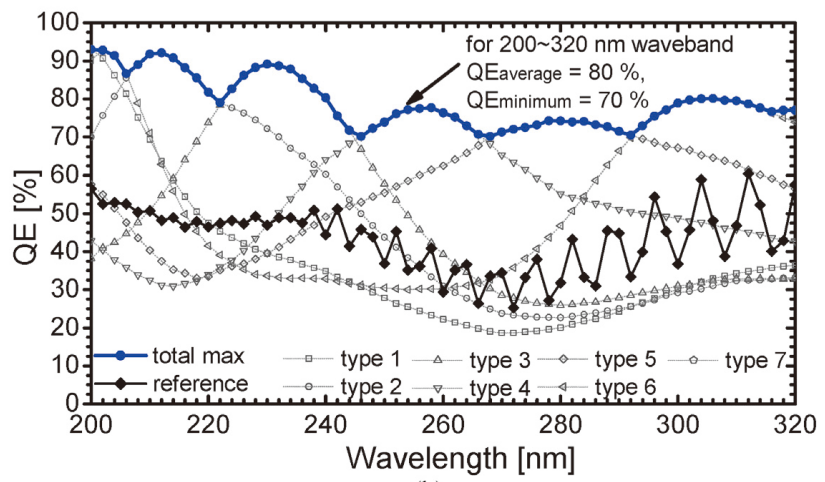

(b)

Fig.8 Measured QE as a function of wavelength of the developed PDA and a reference for (a) 200-1100 nm UV-VIS-NIR waveband and (b) 200-320 nm UV waveband. 


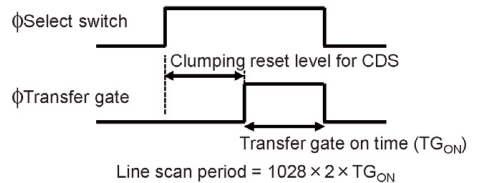

(a)

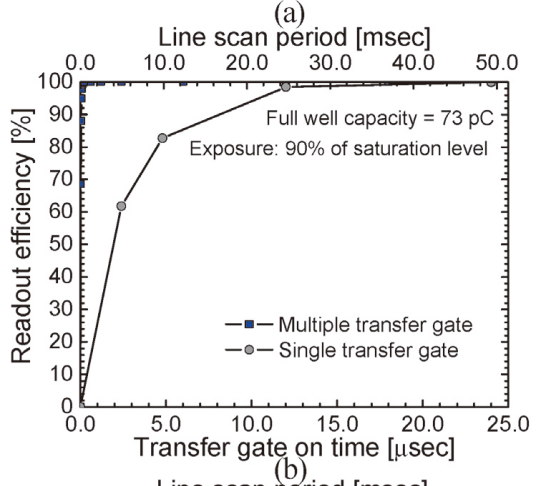

(b)

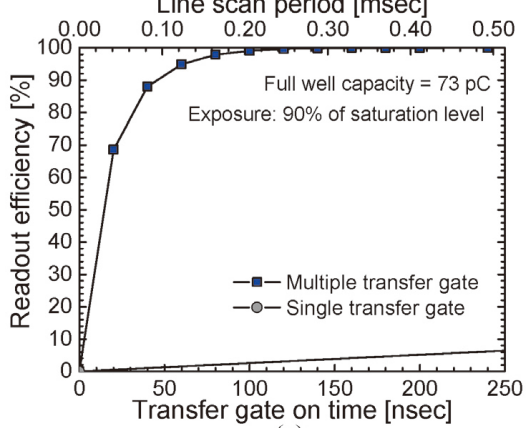

(c)

Fig.9 (a) Diagram of pixel driving pulse, (b) and (c) PD photo charge readout efficiency as a function of transfer gate on time for the developed PDA with multiple transfer gates along the long side of PD and the reference PDA, where (c) is the enlarged view of (b). The line scan period was formulated considering the charge transfer time and correlated double sampling of the charge amplifier.

\subsection{Stability toward Deuterium Lamp Irradiation}

In this subsection, the dopant concentration condition to achieve high stability toward deuterium lamp irradiation is discussed. Regarding the PD technology introduced in the developed PDA in this work that utilizes the surface high concentration layer with steep dopant concentration profile on flattened $\mathrm{Si}$, the stabilities of sensitivity and dark current toward super high pressure mercury lamp were previously evaluated and studied in detail ${ }^{4) ~ 9)}$. It has been clarified that the surface dopant concentration at the top few nanometer Si surface to form a thin neutral region plays a critical role to passivate the interface states and suppress the band bending in the surface region of Si due to the electric flux of the increased fixed charges ${ }^{5), 8)}$. Deuterium lamps have different spectral distribution from super high pressure mercury lamps, and they are widely employed in various spectrometers. The degree of impact to the stability of PD's performances may be

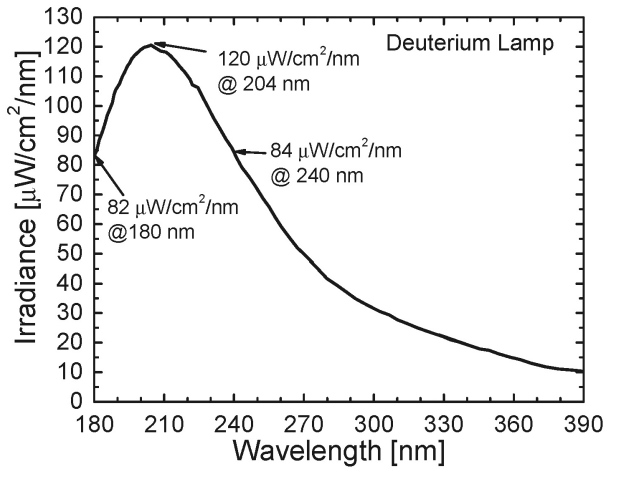

Fig.10 Spectral distribution of the deuterium lamp employed for the stability evaluation.

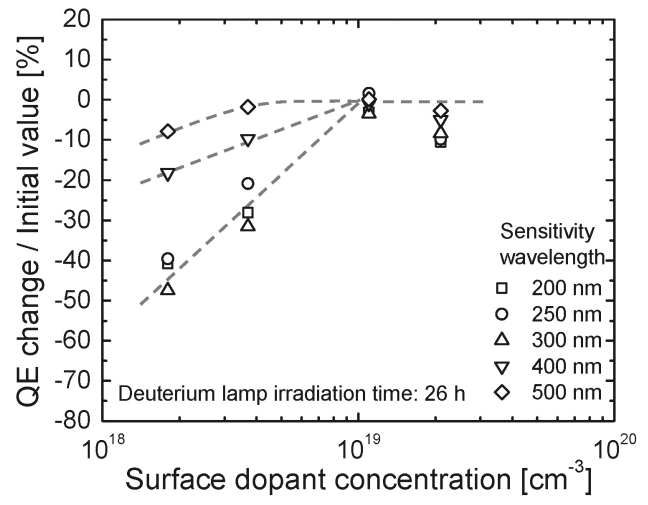

Fig.11 Measured changes of $Q E$ from the initial values after the deuterium lamp irradiation time of $26 \mathrm{~h}$ as a function of surface dopant concentration of surface $\mathrm{p}^{+}$layer.

different from those caused by the super high pressure mercury lamp. In order to clarify the dopant concentration condition to achieve a high stability of light sensitivity, $\mathrm{p}^{+} \mathrm{np}$ PDs with four $\mathrm{p}^{+}$layer formation conditions were fabricated and evaluated. The top surface dopant concentrations of the fabricated four PDs were $1.8 \times 10^{18}, 3.7 \times 10^{18}, 1.1 \times 10^{19}, 2.1 \times 10^{19} \mathrm{~cm}^{-3}$, respectively. Fig.10 shows the spectral distribution of the deuterium lamp, Hamamatsu L8518, employed for the UV-light irradiation stress. It exhibits the peak light intensity at around $204 \mathrm{~nm}$. Fig.11 shows the measured changes of $\mathrm{QE}$ from the initial values after the deuterium lamp irradiation time of $26 \mathrm{~h}$ as a function of the top surface dopant concentration of surface $\mathrm{p}^{+}$layer. The result shows that for the PDs with surface dopant concentration below $10^{19} \mathrm{~cm}^{-3}$, degradations of $\mathrm{QE}$ occurred especially in the UV-light waveband. For light sensitivity wavelength longer than $400 \mathrm{~nm}$, degradation levels decreased. The degradation levels of $\mathrm{QE}$ for all of the measured wavelengths decreased as the surface dopant concentration increased. For the surface dopant concentration higher than $10^{19} \mathrm{~cm}^{-3}$, the degradation 


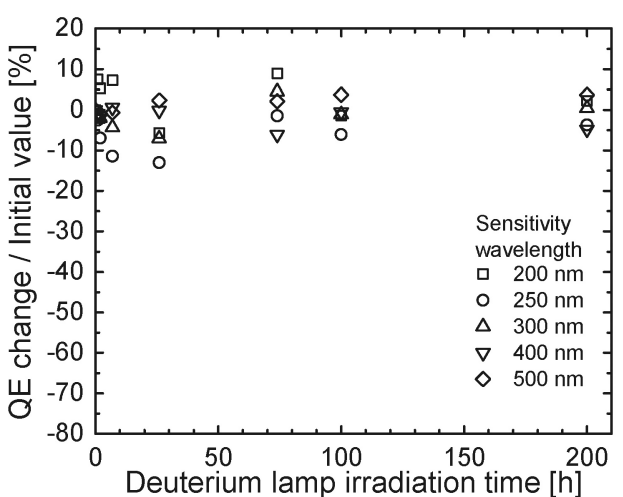

(a)

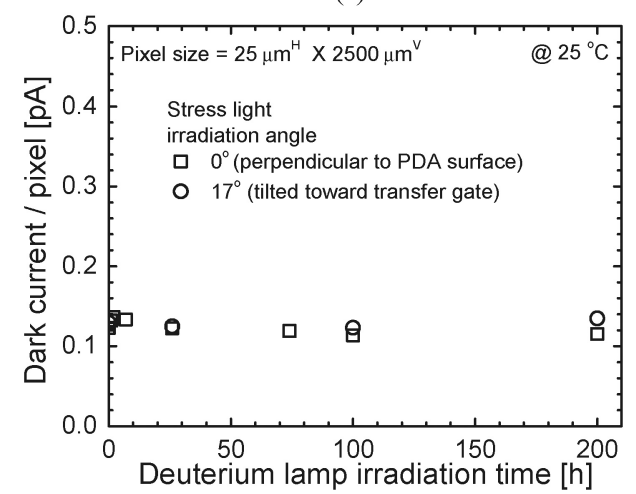

(b)

Fig.12 (a) change of QE for various wavelength normalized by the initial values and (b) dark current as functions of deuterium lamp irradiation time, respectively.

levels were very small. From the obtained result, the surface dopant concentration value must be higher than $10^{19} \mathrm{~cm}^{-3}$ to achieve high stability to the deuterium lamp irradiation stress. For the developed PDA, the surface dopant concentration of the surface $\mathrm{p}^{+}$layer was $1.1 \times$ $10^{19} \mathrm{~cm}^{-3}$. Fig.12 shows the stability of QE and dark current toward the deuterium lamp irradiation. The stabilities after the irradiation time of up to $200 \mathrm{~h}$ were evaluated. The total amount of the light exposure after $200 \mathrm{~h}$ was 59,86 and $60 \mathrm{~J} / \mathrm{cm}^{2}$ for the wavelength of 180 , 204 and $240 \mathrm{~nm}$, respectively. It is sufficiently long for the evaluation of performance stability for general spectrometers over 10 years. In Fig.12 (b), the obtained results under two stress light irradiation angles of $0^{\circ}$ (perpendicular to PDA surface) and $17^{\circ}$ (tilted toward transfer gate) are shown. Here, for the spectrometer usage, light irradiation angles on PDA are not always $0^{\circ}$. Since the surface high concentration $\mathrm{p}^{+}$layer is not formed near the end of the transfer gate side, tilted irradiation angle condition is expected to be severer for the developed PDA. Fig.12 shows that the degradations of $\mathrm{QE}$ and dark current were very small for the all applied stress conditions due to the introduced PD junction technology. The high stability of both $\mathrm{QE}$ and dark current were achieved for the developed PDA.
Table 2 Detail of the seven types of high transmittance optical layers formed on pixel region of the developed PDA.

\begin{tabular}{|c|c|c|}
\hline \multicolumn{2}{|c|}{ Process technology } & $\begin{array}{l}0.18 \mu \mathrm{m} \text { 1P3M CMOS with } \\
\text { buried partially depleted PD }\end{array}$ \\
\hline \multicolumn{2}{|c|}{ Supply voltage } & $3.3 \mathrm{~V}$ \\
\hline \multicolumn{2}{|l|}{ Die size } & $28.6 \mathrm{~mm}^{\mathrm{H}} \times 3.3 \mathrm{~mm}^{\mathrm{V}}$ \\
\hline \multicolumn{2}{|l|}{ Pixel size } & $25 \mu \mathrm{m}^{\mathrm{H}} \times 2500 \mu \mathrm{m}^{\mathrm{V}}$ \\
\hline \multirow{2}{*}{$\begin{array}{l}\text { Number of } \\
\text { pixels }\end{array}$} & total & 1028 \\
\hline & effective & 1024 \\
\hline \multicolumn{2}{|c|}{ Spectral sensitivity range } & $200 \sim 1000 \mathrm{~nm}$ \\
\hline \multirow{2}{*}{\multicolumn{2}{|c|}{$\mathrm{QE}_{\text {average }}$ and $\mathrm{QE}_{\text {minimum }}$}} & $70 \%$ and $50 \%$ for $200 \sim 800 \mathrm{~nm}$ \\
\hline & & $80 \%$ and $70 \%$ for $200 \sim 320 \mathrm{~nm}$ \\
\hline \multicolumn{2}{|c|}{ Full well capacity } & $73 \mathrm{pC}$ \\
\hline \multicolumn{2}{|c|}{$\begin{array}{l}\text { Minimum transfer gate on } \\
\text { time }(\text { lag }<0.1 \%)\end{array}$} & $\begin{array}{l}160 \mathrm{nsec} \\
\text { (line scan period: } 0.33 \mathrm{msec} \text { ) }\end{array}$ \\
\hline \multicolumn{2}{|l|}{ Dark current } & $0.14 \mathrm{pA} /$ pixel \\
\hline
\end{tabular}

Table 2 summarizes the developed PDA chip design specifications and measured performances. The superior performances of the developed PDA chip were successfully confirmed.

\section{Conclusions}

In this paper, a high $\mathrm{QE}$ and high readout speed 1024 pixel UV-VIS-NIR waveband PDA with high transmittance optical layers for multiply divided pixel groups and multiple transfer gates along the long side of PD was designed, fabricated and evaluated. The very high average QE of $70 \%$ for 200-800 $\mathrm{nm}$ and $80 \%$ for 200-320 nm wavebands were successfully obtained. A high FWC of over $70 \mathrm{pC}$ and a high line scan period of $0.33 \mathrm{msec}$ were achieved simultaneously. In addition, it was clarified that the surface dopant concentration must be higher than $10^{19} \mathrm{~cm}^{-3}$ in order to achieve high stability toward deuterium lamp irradiation. The high stabilities of sensitivity and dark current of the fabricated PDA toward deuterium lamp irradiation were successfully confirmed. The developed PDA is promising for performance improvement of UV-VIS-NIR spectrometers. Furthermore, the developed optical layer formation technology is expected to be useful for developments of various types of spectral image sensors.

\section{Acknowledgement}

This work was supported by JST SENTAN-project.

\section{References}

1) R.J. Robbins, S.R. Bean: "Development of a quantitative highperformance liquid chromatography-photodiode array detection measurement system for phenolic acids", J. Chromatography A, 1038, pp.97-105 (June 2004)

2) X. Hou, B.T. Jones, R.A. Meyers: "Inductively Coupled Plasma/Optical Emission Spectrometry", Encyclopedia of 
Analytical Chem., John Wiley \& Sons Ltd, Chichester, pp.94689485 (2000)

3) Y. Gaillard and G. Prpin: "Screening and identification of drugs in human hair by high-performance liquid chromatography-photodiode-array UV detection and gas chromatography-mass spectrometry after solid-phase extraction A powerful tool in forensic medicine", J. Chromatography A, 762, pp.251-267 (Feb. 1997)

4) R. Kuroda, T. Nakazawa, K. Hanzawa and S. Sugawa: "Highly Ultraviolet Light Sensitive and Highly Reliable Photodiode with Atomically Flat Si Surface", Intl. Image Sensor Workshop, pp.3841 (June 2011)

5) T. Nakazawa, R. Kuroda, Y. Koda and S. Sugawa: "Photodiode dopant structure with atomically flat $\mathrm{Si}$ surface for high sensitivity to UV-light", SPIE-IS\&T, 8298, pp.82980M-1-8 (Feb. 2012)

6) T. Akutsu, S. Kawada, Y. Koda, T. Nakazawa, R. Kuroda and S. Sugawa: "A 1024 x 1 Linear Photodiode Array with Fast Readout Speed Flexible Pixel-level Integration Time and High Stability to UV Light Exposure", IS\&T/SPIE EI, 9022, pp.90220L-1-8 (Feb. 2014)

7) S. Nasuno, S. Kawada, Y. Koda, T. Nakazawa, K. Hanzawa, R. Kuroda and S. Sugawa: "A wide dynamic range CMOS image sensor with 200-1100 nm spectral sensitivity and high robustness to UV right exposure", Jpn.J. Appl. Phys., 53, pp.04EE07-1-4 (Mar. 2014)

8) R. Kuroda, S. Kawada, S. Nasuno, T. Nakazawa, Y. Koda, K. Hanzawa and S. Sugawa: "A Highly Ultraviolet Light Sensitive and Highly Robust Image Sensor Technology Based on Flattened Si Surface", ITE Trans. MTA, 2, pp.123-130 (Apr. 2014)

9) R. Kuroda and S. Sugawa: "Si image sensors with wide spectral response and high robustness to ultraviolet light exposure", IEICE Electronics Express, 11, pp.20142004-1-16 (May 2014)

10) A. Lambrechts, P. Gonzalez, B. Geelen, P. Soussan, K. Tack and M. Jayapala: "A CMOS-compatible, integrated approach to hyperand multispectral imaging", Int. Electron Device Meeting 2014, pp.261-264 (Dec. 2014)

11) Y. Koda, R. Kuroda and S. Sugawa: "High quantum efficiency 200$1000 \mathrm{~nm}$ spectral response photodiodes with on-chip multiple high transmittance optical layers", in Proc. IEEE Sensors 2014, pp.1664-1667 (Nov. 2014)

12) R. Kuroda, T. Akutsu, Y. Koda, K. Takubo, H. Tominaga, R. Hirose, T. Karasawa and S. Sugawa: "A 80\% QE High Readout Speed 1024 Pixel Linear Photodiode Array for UV-VIS-NIR Spectroscopy", Intl. Image Sensor Workshop, pp.78-81 (June 2015)

13) K. Koda, R. Kuroda, T. Nakazawa, S. Sugawa: "A UV Siphotodiode with almost $100 \%$ internal Q.E. and high transmittance on-chip multilayer dielectric stack", IS\&T/SPIE EI, 8659, pp.86590J-1-6 (Feb. 2013)

14) G. Santana and A.M. Acevedo: "Optimization of PECVD SiH:H films for silicon solar cells", Solar Energy Materials \& Solar cells, 60, pp.135-142 (2000)

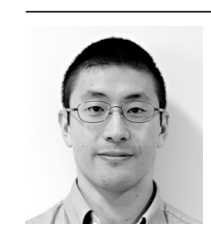

Rihito Kuroda received the B.S. degree in electronic engineering and the M.S. and Ph.D. degrees in management science and technology from Tohoku University, Sendai, Japan, in 2005, 2007, and 2010, respectively. He was a Research Fellow of the Japan Society for the Promotion of Science Research from 2007 to 2010. Since 2010, he is with the Graduate School of Engineering, Tohoku University, where he is currently an Associate Professor.

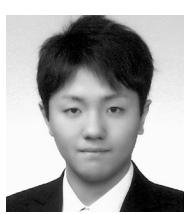

Takahiro Akutsu received the B.S. degree in electronic engineering and M.S. degree in management science and technology from Tohoku University, Sendai, Japan, in 2013 and 2015, respectively. He is currently with the Central Japan Railway Company.

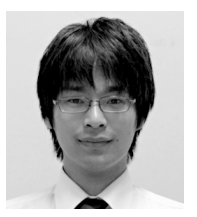

Yasumasa Koda received the B.S. degree in electronic engineering and M.S. degree in management science and technology from Tohoku University, Sendai, Japan, in 2012 and 2014, respectively. He is currently pursuing the $\mathrm{Ph} . \mathrm{D}$. degree with the Graduate School of Engineering, Tohoku University.

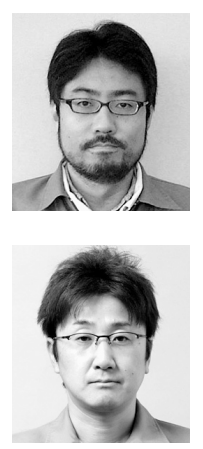

Kenji Takubo received the M.S. degree in electrical engineering from Kyoto University, Kyoto, Japan, in 1992. He joined Shimadzu Corporation, Kyoto, Japan, in 1992, where he is engaged in the development of optical sensors and analytical instruments.

Hideki Tominaga received the M.S. degree in science engineering from Hiroshima University, Hiroshima, Japan, in 1991. He joined Shimadzu Corporation, Kyoto, Japan, in 1991, where he is engaged in the development of high-speed video camera systems.

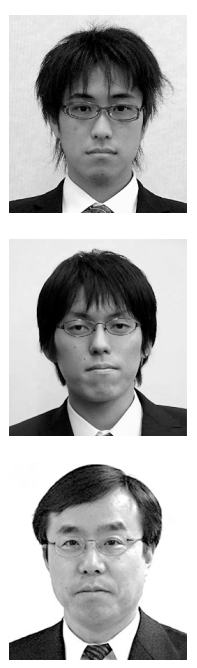

Ryuta Hirose received the M.S. degree in electrical engineering from Kyoto institute of Technology, Kyoto, Japan, in 2007. He joined Shimadzu Corporation, Kyoto, Japan, in 2007, where he is engaged in the development of optical sensors.

Tomohiro Karasawa received the M.S. degree in engineering from Tsukuba University, Ibaraki, Japan, in 2012. He joined Shimadzu Corporation, Kyoto, Japan, in 2012, where he is engaged in the development of optical sensors.

Shigetoshi Sugawa received the M.S. degree in physics from the Tokyo Institute of Technology, Tokyo, Japan, in 1982 and the Ph.D. degree in electrical engineering from Tohoku University, Sendai, Japan, in 1996. In 1982-1999, he was with Canon Inc. In 1999, he moved to Tohoku University, where he is currently a Professor with the Graduate School of Engineering, also with the New Industry Creation Hatchery Center. ITE fellow. 